\title{
Synthesis of Borylated Hydrazino Acid Derivatives
}

\section{Chieh-Hung Tien \\ Alina Trofimova \\ Wenjie Shao \\ Andrei K. Yudin*}

Davenport Research Laboratories, Department of Chemistry, University of Toronto, 80 St. George Street, Toronto, ON M5S 3H6, Canada

andrei.yudin@utoronto.ca

Published as part of the

Special Issue dedicated to Prof. Sarah Reisman, recipient of the 2019
Dr. Margaret Faul Women in Chemistry Award

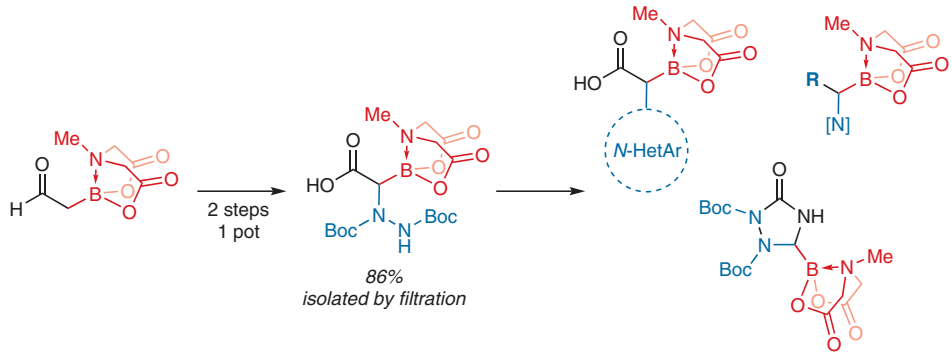

Received: 04.04.202

Accepted after revision: 07.05.2021

Published online: 31.05 .2021

DOI: 10.1055/s-0040-1706046; Art ID: ss-2021-t0192-st

Abstract $\alpha$-Boryl- $\alpha$-hydrazinoacetic acid is a highly functionalized boron-containing building block that can be easily accessed from readily available $\alpha$-borylacetaldehyde. The hydrazine motif can be converted into a variety of $\alpha$-borylated azoles and diazines in a straightforward protocol. Furthermore, the carboxy group can be derivatized to afford novel organoboron compounds that should find applications in various cross-coupling transformations.

Key words hydrazinoboronates, aminoboronic acids, organoboron, borylated heterocycles, BMIDA

Organoboron compounds have found widespread applications in various fields of inquiry. ${ }^{1}$ The ability of boron to reversibly bind to heteroatom nucleophiles, combined with boric acid's low toxicity (main metabolite of organoboron compounds; $\mathrm{LD}_{50}$ comparable to table salt) have allowed the identification of numerous boron-containing molecules as promising candidates in drug discovery. ${ }^{1 \mathrm{~d}, 2}$ Among the many boron-containing scaffolds, borylated peptidomimetics derived from $\alpha$-aminoboronic acids often display potent antitumor properties. ${ }^{3}$ As such, the synthesis of $\alpha$-aminoboronic acids has been a subject of ongoing interest (Scheme 1). ${ }^{4}$

Compared to $\alpha$-aminoboronic acids, $\alpha$-hydrazinoboronic acids have received no attention despite the prevalence of hydrazine building blocks in organic chemistry. ${ }^{5}$ The hydrazine motif is frequently employed in the synthesis of heterocyclic scaffolds with biomedical applications. ${ }^{6}$ In addition to being versatile synthetic handles, incorporation of the hydrazine functional group in peptidic scaffolds results in aza- $\beta$-peptides, which can display new secondary structures when compared to the parental forms. ${ }^{7}$ As part of our ongoing efforts in the pursuit of unique boron-containing building blocks, ${ }^{8}$ we report the synthesis and utility of $\alpha$-boryl- $\alpha$-hydrazinoacetic acid $\mathbf{1}$.

Treatment of readily available $\alpha$-borylacetaldehyde $\mathbf{2}^{8 \mathrm{a}}$ with DBAD (di-tert-butyl azodicarboxylate) in the presence of catalytic amount of proline yielded $\alpha$-boryl- $\alpha$-hydrazinoacetaldehyde $\mathbf{3}$ with complete consumption of $\mathbf{2}$ (Scheme 2). ${ }^{9}$ No side products were generated based on ${ }^{1} \mathrm{H}$ NMR
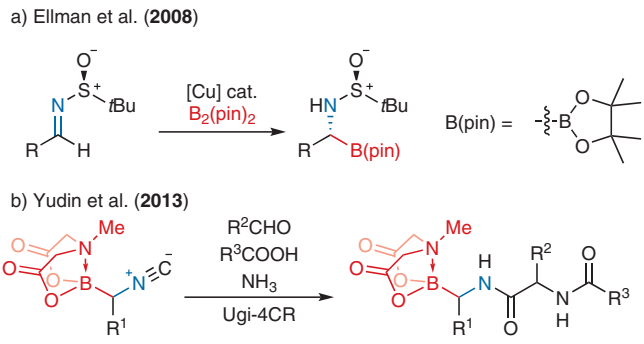

c) Yudin et al. (2016) and Bode et al. (2018)

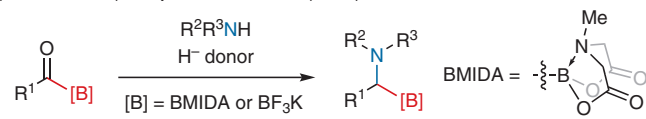

d) Sawamura et al. (2019)

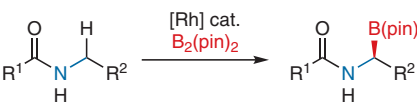

e) This work: Synthesis of $\alpha$-boryl- $\alpha$-hydrazinoacetic acid (1)

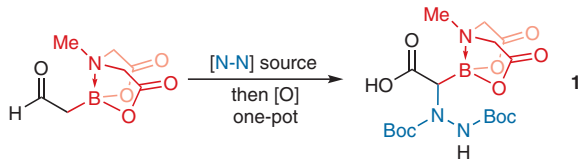

Scheme 1 Synthesis of $\alpha$-aminoboronates: (a) Cu-catalyzed borylation of enantiopure sulfinyl imines. (b) Ugi 4C reaction using $\alpha$-boryl isocyanides with aldehydes, carboxylic acids, and ammonia. (c) Reductive amination of MIDA acyl boronates and potassium acyltrifluoroborates. (d) Rh-catalyzed amine-directed $\mathrm{C}\left(\mathrm{sp}^{3}\right)-\mathrm{H}$ borylation. (e) Synthesis of $\alpha$-boryl- $\alpha$-hydrazinoacetic acid 1 . 
spectroscopy of the crude reaction mixture. Accordingly, we moved forward with the reaction by carrying out a Pinnick oxidation. ${ }^{8 a}$ To our delight, highly functionalized MIDA boronate 1 was isolated by filtration in $86 \%$ yield over two steps in a one-pot fashion.

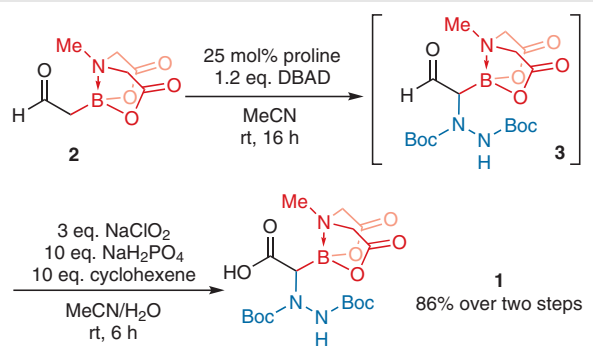

Scheme 2 Synthesis of MIDA boronate 1 via a proline-catalyzed $\alpha$-amination of $\alpha$-borylacetaldehyde 2 then subsequent Pinnick oxidation in one pot

With 1 in hand, we set out to explore the utility of the hydrazine moiety. Deprotection of the Boc (tert-butoxycarbonyl) group in a DCM/TFA mixture provided the free hydrazine salt 4 in quantitative yield after 30 minutes without any signs of MIDA deprotection or protodeboronation. Upon evaporation of the solvent, reaction with 1,3- or 1,4electrophiles in $\mathrm{MeCN}$ yielded a series of $N$-heterocyclesubstituted $\alpha$-carboxy MIDA boronates 5 (Scheme 3). Reaction with acetylacetaldehyde dimethyl acetal produced a 4:1 mixture of 1,5- and 1,3-substituted pyrazole $\mathbf{5 a}$ in quantitative yield. Condensation with acetylacetone and dibenzoylmethane gave $\mathbf{5 b}$ and $\mathbf{5 c}$, respectively. However, the reaction to give $\mathbf{5 c}$ did not go to completion after $20 \mathrm{~h}$, presumably due to reduced reactivity of dibenzoylmethane. Aryl keto-enamines reacted efficiently with $\mathbf{4}$ to form the corresponding 5-arylpyrazoles in high yields (5d-5f). Cyclization with arylated malondialdehydes successfully gave the corresponding 4-arylpyrazoles (5g and $\mathbf{5 h}$ ). Pyrazolone 5i was generated when ethyl acetoacetate was used as the bis-electrophile. Similarly, methyl 2-formylbenzoate and 2formylphenylboronic acid were suitable 1,4-electrophiles under these conditions and the reactions provided phthalazinone 5j and 1,2,3-diazaborinine 5k, respectively. Lastly, condensation with phthalic anhydride afforded a mixture of phthalazinedione $\mathbf{5 l}$ and $\mathrm{N}$-aminophthalimide $\mathbf{5 l}$ ' which are separable by column chromatography.

We next turned our attention towards the derivatization of the carboxy functional group (Scheme 4). Methylation of the carboxylic acid with $\mathrm{TMSCHN}_{2}$ gave the methyl ester in good yield (6a). Activation of the acid with HATU and subsequent reduction of the activated ester with $\mathrm{NaBH}_{4}$ gave $\beta$-hydrazino alcohol $\mathbf{6 b}$. Similarly, borylated oxadiazole $\mathbf{6 c}$ could be synthesized using a one-pot HATU-mediated coupling protocol adapted from our previous report. ${ }^{8 \mathrm{e}}$ Activation of the carboxylic acid with DIC in the presence of 4-nitrobenzyl alcohol provided the corresponding 4-nitro-
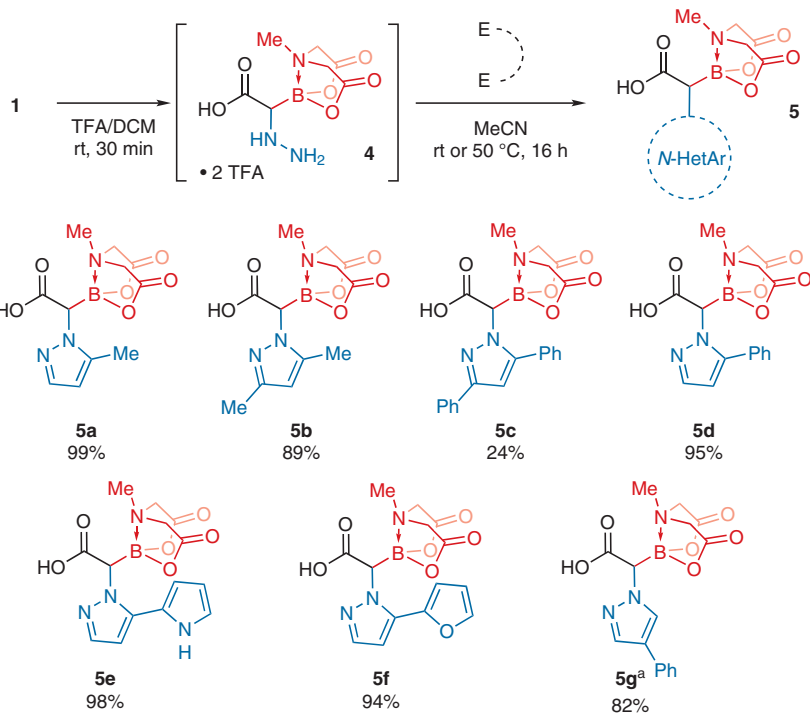

$5 b$
$89 \%$

$5 c$
$24 \%$

$5 d$
$95 \%$
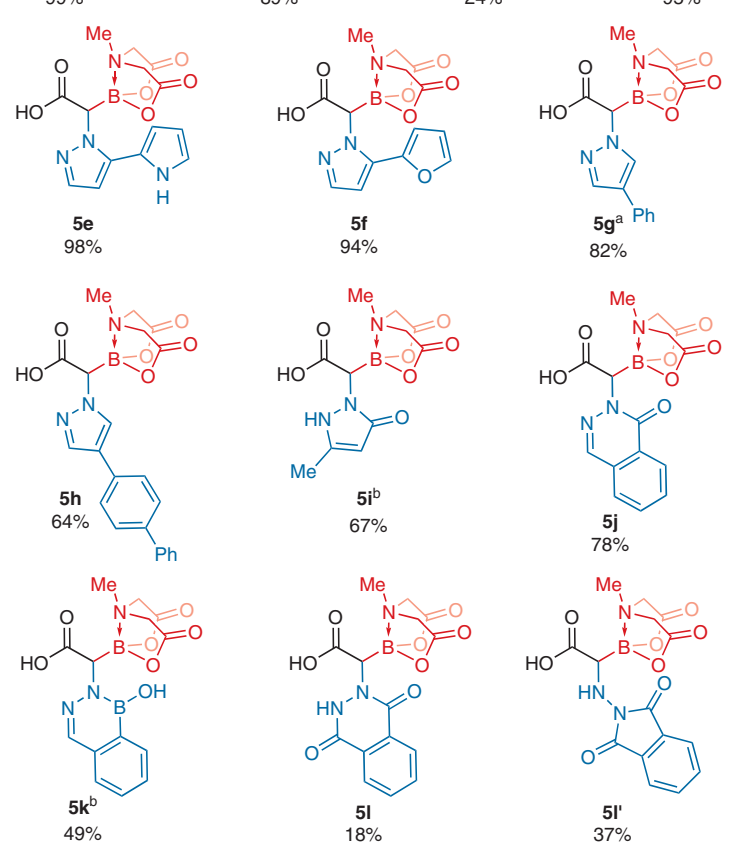

Scheme 3 Scope of $N$-heterocycle substituted $\alpha$-carboxy MIDA boronates from the condensation of $\mathbf{4}$ with a variety of electrophilic accep-

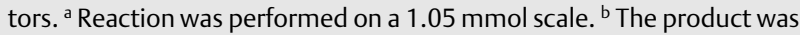
isolated as a TFA salt.

benzyl ester $\mathbf{6 d}$ in excellent yield. ${ }^{10}$ Additionally, redox-active ester $\mathbf{6 e}$ could be synthesized on a $1-\mathrm{g}$ scale in good yield using $N$-hydroxyphthalimide as the nucleophile. Reaction with DPPA (diphenylphosphoryl azide) under mild heat provided triazolidinone $\mathbf{6 f}$ via the Curtius rearrangement. This represents one of the few examples of a boronate that is attached to a carbon atom with the oxidation state of an aldehyde. ${ }^{11}$ Subsequent $N$-alkylation of $\mathbf{6 f}$ with 4-nitrobenzyl bromide gave $\mathbf{6 g}$, the structure of which was confirmed by single crystal X-ray diffraction (Figure 1).

In conclusion, we have developed a straightforward synthesis of $\alpha$-carboxy- $\alpha$-hydrazino MIDA boronate $\mathbf{1}$ using a column-free protocol from readily available aldehyde $\mathbf{2}$. The highly functionalized building block was used to access a variety of boro-methylated heterocycles and carboxy derivatives. The resulting borylated compounds $\mathbf{5}$ and $\mathbf{6}$ have 


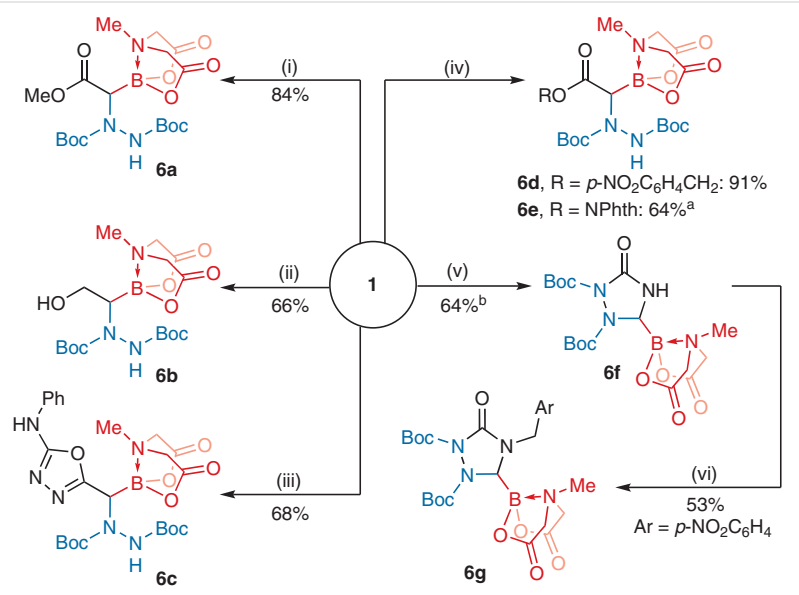

Scheme 4 Functionalization of the carboxy moiety of 1. Reagents and conditions: (i) $\mathrm{TMSCHN}_{2}$ (2 equiv), THF/MeOH then $\mathrm{AcOH}, 84 \%$; (ii) HATU ( 1.2 equiv), DIPEA (3 equiv), MeCN then $\mathrm{NaBH}_{4}$ ( 3 equiv), $66 \%$; (iii) HATU (1.1 equiv), DIPEA (3 equiv), PhNHC(S) $\mathrm{NHNH}_{2}$ (1.1 equiv), $\mathrm{MeCN}$ then $\mathrm{TsCl}$ (3.2 equiv) and DIPEA (3 equiv), 68\%; (iv) DIC (1.1-1.2 equiv), DMAP (10 mol\%), ROH (1.1-1.2 equiv), DCM/MeCN, 91\% and 64\%; (v) DPPA (1.1 equiv), DIPEA (2.2 equiv), MeCN, 64\%; (vi) 4-nitrobenzyl bromide ( 1.2 equiv), $\mathrm{K}_{2} \mathrm{CO}_{3}$ ( 2 equiv), TBAB (10 mol\%),

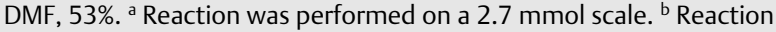
was performed on a $5.4 \mathrm{mmol}$ scale.

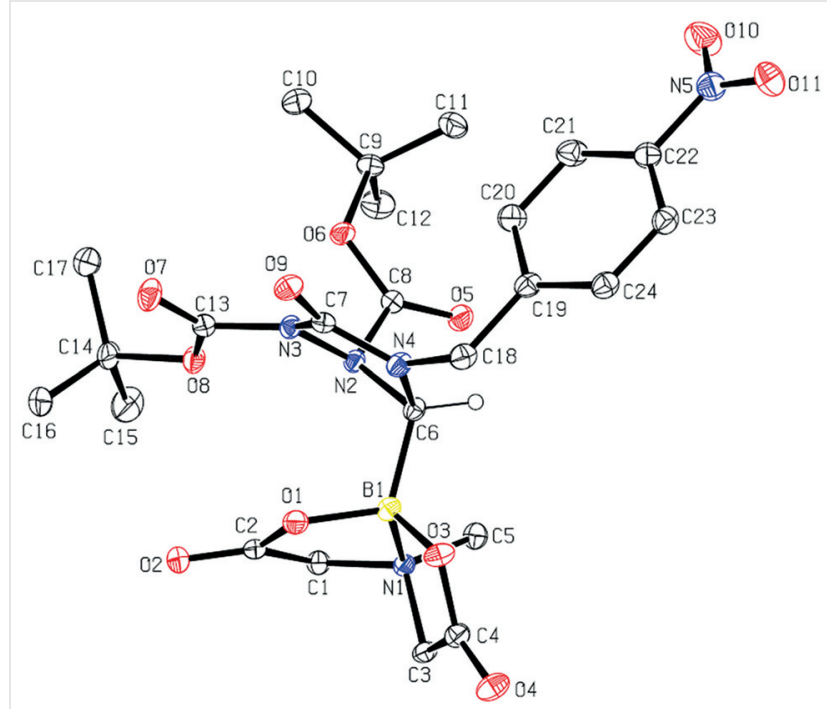

Figure 1 X-ray crystal structure of $\mathbf{6 g}$. Thermal ellipsoid probabilities set at $50 \%$. Hydrogen atoms are omitted for clarity.

considerable potential in transition-metal-catalyzed SuzukiMiyaura cross-coupling and decarboxylative cross-coupling to access value-added products.
All solvents and reagents were purchased from commercial sources and used as received unless otherwise stated. HPLC grade MeCN, hexane, and $i$-PrOH were purchased from MilliporeSigma and used as received. THF was distilled from Na benzophenone ketyl prior to use. Aryl keto-enamine, ${ }^{12}$ malondialdehydes, ${ }^{13}$ and $\mathbf{2}^{8 \mathrm{c}}$ were synthesized according to literature procedures. Flash column chromatography was carried out using Silicycle 230-400 mesh silica gel. Thin-layer chromatography was performed on Merck Aluminum-backed TLC Silica gel $60 \mathrm{~F}^{254}$ and visualized using a UV lamp (254 nm) and curcumin stain. Reverse-phase chromatography was carried out using Biotage SNAP ultra C18 on a Teledyne-Isco Combiflash system. ${ }^{1} \mathrm{H},{ }^{11} \mathrm{~B},{ }^{13} \mathrm{C}$, and ${ }^{19} \mathrm{~F}$ NMR spectra were recorded on Bruker $400 \mathrm{MHz}$ and $500 \mathrm{MHz}$ spectrometers at $23{ }^{\circ} \mathrm{C}$ unless otherwise stated. ${ }^{1} \mathrm{H}$ NMR chemical shifts are referenced to residual protonated solvent peak $\left(\mathrm{MeCN}-d_{3}: \delta\right.$ $=1.94 ;$ DMSO- $\left.d_{6}: \delta=2.50\right) .{ }^{11} \mathrm{~B}$ NMR chemical shifts are referenced to an external standard of $\mathrm{BF}_{3} \cdot \mathrm{OEt}_{2}(\delta=0.0) .{ }^{13} \mathrm{C}$ NMR chemical shifts are referenced to the corresponding solvent peaks $\left(\mathrm{MeCN}-d_{3}: \delta=118.2\right.$; DMSO- $d_{6}: \delta=39.5$ ). Carbon atoms exhibiting significant line broadening brought about by boron substituents were not reported due to quadrupolar relaxation. ${ }^{19} \mathrm{~F}$ NMR chemical shifts are referenced to an external standard of $\mathrm{CFCl}_{3}(\delta=0.0)$. HRMS were obtained on a VG 70$250 \mathrm{~S}$ (double focusing) mass spectrometer at $70 \mathrm{eV}$ or on an ABI/Sciex Qstar mass spectrometer with ESI or DART sources, MS/MS and accurate mass capabilities. Low-resolution mass spectra (ESI) were collected on an Agilent Technologies 1200 series HPLC paired to a 6130 Mass Spectrometer. FTIR analysis was carried out on a Bruker Alpha Platinum ATP spectrometer and peaks below $1500 \mathrm{~cm}^{-1}$ are not reported. Melting points were measured uncorrected utilizing a MelTemp capillary melting point apparatus.

\section{$\alpha$-Boryl-a-hydrazinoacetic Acid 1}

To a stirred suspension of $\mathbf{2}$ ( $1.00 \mathrm{~g}, 5.03 \mathrm{mmol}, 1$ equiv) in $\mathrm{MeCN}$ (50 $\mathrm{mL})$ were added di-tert-butyl azodicarboxylate $(1.39 \mathrm{~g}, 6.03 \mathrm{mmol}$, 1.2 equiv) and L-proline ( $145 \mathrm{mg}, 1.26 \mathrm{mmol}, 25 \mathrm{~mol} \%$ ). The mixture was stirred at rt for $16 \mathrm{~h}$. Cyclohexene ( $5.1 \mathrm{~mL}, 50.3 \mathrm{mmol}, 10$ equiv), $\mathrm{NaH}_{2} \mathrm{PO}_{4} \cdot \mathrm{H}_{2} \mathrm{O}$ (6.94 g, $50.3 \mathrm{mmol}, 10$ equiv), $\mathrm{NaClO}_{2}$ (1.36 g, 15.1 mmol, 3 equiv), and water $(30 \mathrm{~mL})$ were subsequently added. The mixture was stirred at $\mathrm{rt}$ for $6 \mathrm{~h}$. Upon completion of reaction as indicated by ${ }^{1} \mathrm{H}$ NMR spectroscopy, the organic layer was removed from the biphasic mixture in vacuo. $\mathrm{MeCN}(10 \mathrm{~mL})$ was added, then the mixture was cooled for $1 \mathrm{~h}$ in a $4{ }^{\circ} \mathrm{C}$ fridge. The mixture was filtered, then the filter cake was washed with water $(20 \mathrm{~mL})$ and $\mathrm{Et}_{2} \mathrm{O}(50 \mathrm{~mL})$ to afford 1 (1.93 g, $4.33 \mathrm{mmol}, 86 \%$ ) as a colorless solid; mp 207-210 ${ }^{\circ} \mathrm{C}$.

IR (neat): 3173, 2979, 1799, 1773, 1726, 1714, 1662, $1524 \mathrm{~cm}^{-1}$.

${ }^{1} \mathrm{H}$ NMR $\left(500 \mathrm{MHz}, 333 \mathrm{~K}\right.$, DMSO- $\left.d_{6}\right): \delta=8.12(\mathrm{br} \mathrm{s}, 1 \mathrm{H}), 4.37(\mathrm{~s}, 1 \mathrm{H})$, $4.27(\mathrm{~d}, J=17.5 \mathrm{~Hz}, 1 \mathrm{H}), 4.21(\mathrm{~d}, J=17.0 \mathrm{~Hz}, 1 \mathrm{H}), 4.00(\mathrm{~d}, J=17.5 \mathrm{~Hz}$, $1 \mathrm{H}), 3.95(\mathrm{~d}, J=17.0 \mathrm{~Hz}, 1 \mathrm{H}), 3.16(\mathrm{~s}, 3 \mathrm{H}), 1.43(\mathrm{~s}, 9 \mathrm{H}), 1.40(\mathrm{~s}, 9 \mathrm{H})$. ${ }^{13} \mathrm{C}$ NMR $\left(126 \mathrm{MHz}, 333 \mathrm{~K}\right.$, DMSO- $\left.d_{6}\right): \delta=173.6,168.0,167.7,80.7$, $80.2,62.7,46.3,27.7,27.5$.

${ }^{11} \mathrm{~B}$ NMR $\left(160 \mathrm{MHz}, 333 \mathrm{~K}, \mathrm{DMSO}-d_{6}\right): \delta=9.8$.

HRMS (ESI): $m / z[\mathrm{M}+\mathrm{Na}]^{+}$calcd for $\mathrm{C}_{17} \mathrm{H}_{28} \mathrm{BN}_{3} \mathrm{NaO}_{10}: 467.1796$; found: 467.1795 .

\section{$\alpha$-Boryl- $\alpha$-hydrazinoacetaldehyde 3}

To a stirred suspension of 2 ( $0.400 \mathrm{~g}, 2.01 \mathrm{mmol}, 1$ equiv) in $\mathrm{MeCN}$ (10 $\mathrm{mL}$ ) were added di-tert-butyl azodicarboxylate $(0.555 \mathrm{~g}, 2.41 \mathrm{mmol}$, 1.2 equiv) and L-proline (58 $\mathrm{mg}, 0.50 \mathrm{mmol}, 25 \mathrm{~mol} \%$ ). The mixture was stirred at $\mathrm{rt}$ for $16 \mathrm{~h}$. The mixture was diluted with MeCN (100 $\mathrm{mL}$ ) and loaded onto Celite. The volatiles were removed in vacuo, then 
the residue was purified by normal-phase column chromatography (mixture hexanes/acetone) to afford $3(0.656 \mathrm{~g}, 1.53 \mathrm{mmol}, 76 \%)$ as a colorless solid; mp $120-122^{\circ} \mathrm{C}$.

IR (neat): 3274, 2932, 2861, 1772, 1717, 1685, $1500 \mathrm{~cm}^{-1}$.

${ }^{1} \mathrm{H}$ NMR $\left(500 \mathrm{MHz}, \mathrm{MeCN}-d_{3}\right): \delta=9.73(\mathrm{~s}, 1 \mathrm{H}), 6.95-7.10(\mathrm{br}, 1 \mathrm{H})$, 4.63-4.78 (br, $1 \mathrm{H}), 3.96-4.09$ (m, $4 \mathrm{H}), 3.22(\mathrm{~s}, 3 \mathrm{H}), 1.41$ (s, $18 \mathrm{H})$.

${ }^{13} \mathrm{C}$ NMR $\left(126 \mathrm{MHz}, \mathrm{MeCN}-d_{3}\right): \delta=203.1,167.7,156.2,156.1,81.4$, 80.7, 63.4, 63.0, 47.0, 27.5, 27.3.

${ }^{11} \mathrm{~B}$ NMR $\left(160 \mathrm{MHz}, \mathrm{MeCN}-d_{3}\right): \delta=10.2$.

HRMS (ESI): $m / z$ [M + Na] $]^{+}$calcd for $\mathrm{C}_{17} \mathrm{H}_{28} \mathrm{BN}_{3} \mathrm{NaO}_{9}: 451.1847$; found: 451.1851 .

\section{Hydrazine Condensation; General Procedure}

To a 1-dram vial containing 1 ( $44.5 \mathrm{mg}, 0.1 \mathrm{mmol}, 1$ equiv) was added DCM $(0.5 \mathrm{~mL})$ and TFA $(0.5 \mathrm{~mL})$. The mixture was stirred for $30 \mathrm{~min}$ at rt. The volatiles were removed in vacuo, then $\mathrm{MeCN}(1 \mathrm{~mL})$ was added. To the stirring solution was added the appropriate electrophile (1-1.1 equiv), then the mixture was stirred at rt or $50{ }^{\circ} \mathrm{C}$ for $16 \mathrm{~h}$. The mixture was loaded onto Celite, then the volatiles were removed in vacuo. The residue was subjected to reversed-phase column chromatography (gradient of water/MeCN 95:5 to 0:100) to afford the corresponding products upon removal of solvents. Alternatively, if the product precipitated from the mixture, then the mixture was filtered, and the filter cake was washed with $\mathrm{MeCN}(1 \mathrm{~mL})$ to afford the corresponding products.

\section{2-(5-Methyl-1H-pyrazol-1-yl)-2-(MIDA-boryl)acetic Acid (5a)}

The reaction was carried out according to the general procedure using acetylacetaldehyde dimethyl acetal $(14.6 \mathrm{~mL}, 0.11 \mathrm{mmol}, 1.1$ equiv $)$ at rt and purified by reversed-phase column chromatography to afford 5a (29.2 mg, $0.099 \mathrm{mmol}, 99 \%)$ as a colorless solid; mp $176-180{ }^{\circ} \mathrm{C}$ (decomp.).

IR (neat): 2982, 1771, 1746, 1717, $1527 \mathrm{~cm}^{-1}$.

${ }^{1} \mathrm{H}$ NMR $\left(500 \mathrm{MHz}, \mathrm{MeCN}-d_{3}\right): \delta=7.44(\mathrm{~d}, J=2.5 \mathrm{~Hz}, 1 \mathrm{H}), 7.35^{*}(\mathrm{~d}, J=$ $1.5 \mathrm{~Hz}), 6.10$ (d, $J=2.0 \mathrm{~Hz}, 1 \mathrm{H}), 6.09^{*}(\mathrm{~d}, J=1.0 \mathrm{~Hz}), 4.98(\mathrm{~s}, 1 \mathrm{H}), 4.89^{*}$ (s), $4.30^{*}(\mathrm{~d}, J=16.5 \mathrm{~Hz}), 4.20(\mathrm{~d}, J=16.0 \mathrm{~Hz}, 1 \mathrm{H}), 4.1-4.02$ (overlapped, $2 \mathrm{H}$ ), 4.08-4.02* (overlapped), 3.12 (s, $3 \mathrm{H}), 3.07^{*}(\mathrm{~s}), 2.23^{*}(\mathrm{~s})$, $2.22(\mathrm{~s}, 3 \mathrm{H})$; * minor 1,3-regioisomer.

${ }^{13} \mathrm{C}$ NMR $\left(126 \mathrm{MHz}, \mathrm{MeCN}-d_{3}\right): \delta=173.2,172.8^{*}, 169.0^{*}, 168.9^{*}, 168.8$, 168.6, 148.7, 141.5* 138.6* 133.6, 106.1 (major and minor), 65.1*, $65.0^{*}, 64.8,64.5,47.9^{*}, 47.9,13.3,11.1^{*}$; * minor regioisomer.

${ }^{11} \mathrm{~B}$ NMR $\left(128 \mathrm{MHz}, \mathrm{MeCN}-d_{3}\right): \delta=10.4$.

HRMS (ESI): $m / z$ [M - H] $]^{-}$calcd for $\mathrm{C}_{11} \mathrm{H}_{13} \mathrm{BN}_{3} \mathrm{O}_{6}$ : 293.0939; found: 293.0944.

\section{2-(3,5-Dimethyl-1H-pyrazol-1-yl)-2-(MIDA-boryl)acetic Acid (5b)}

The reaction was carried out according to the general procedure using acetylacetone ( $11.2 \mu \mathrm{L}, 0.11 \mathrm{mmol}, 1.1$ equiv) at rt and purified by reversed-phase column chromatography to afford $\mathbf{5 b}(27.4 \mathrm{mg}$, $0.089 \mathrm{mmol}, 89 \%$ ) as a colorless solid ; $\mathrm{mp} 110-115^{\circ} \mathrm{C}$ (decomp.).

IR (neat): 2968, 2551, 1768, 1669, 1586, $1520 \mathrm{~cm}^{-1}$.

${ }^{1} \mathrm{H}$ NMR $\left(500 \mathrm{MHz}, \mathrm{MeCN}-d_{3}\right): \delta=5.85(\mathrm{~s}, 1 \mathrm{H}), 4.76(\mathrm{~s}, 1 \mathrm{H}), 4.27(\mathrm{~d}, J=$ $16.5 \mathrm{~Hz}, 2 \mathrm{H}$ ), 4.08-3.99 (m, $3 \mathrm{H}$ ), 3.07 (s, $3 \mathrm{H}$ ), 2.14 (s, $3 \mathrm{H}), 2.12$ (s, 3 $\mathrm{H})$.
${ }^{13} \mathrm{C}$ NMR $\left(126 \mathrm{MHz}, \mathrm{MeCN}-d_{3}\right): \delta=173.4,169.1,168.9,147.7,142.3$, 105.8, 65.1, 65.0, 48.0, 13.4, 11.1 .

${ }^{11} \mathrm{~B}$ NMR $\left(128 \mathrm{MHz}, \mathrm{MeCN}-d_{3}\right): \delta=10.6$.

HRMS (ESI): $m / z[\mathrm{M}-\mathrm{H}]^{-}$calcd for $\mathrm{C}_{12} \mathrm{H}_{15} \mathrm{BN}_{3} \mathrm{O}_{6}$ : 307.1096; found: 307.1097.

\section{2-(3,5-Diphenyl-1H-pyrazol-1-yl)-2-(MIDA-boryl)acetic Acid (5c)}

The reaction was carried out according to the general procedure using 1,3-diphenylpropane-1,3-dione ( $14.6 \mu \mathrm{L}, 0.11 \mathrm{mmol}, 1.1$ equiv) at 50 ${ }^{\circ} \mathrm{C}$ and purified by reversed-phase column chromatography to afford 5c (10.2 mg, $0.024 \mathrm{mmol}, 24 \%$ ) as a colorless solid; mp 208-213 ${ }^{\circ} \mathrm{C}$.

IR (neat): 2968, 1772, 1766, 1607, $1557 \mathrm{~cm}^{-1}$.

${ }^{1} \mathrm{H}$ NMR $\left(500 \mathrm{MHz}, \mathrm{MeCN}-d_{3}\right): \delta=7.80-7.78(\mathrm{~m}, 2 \mathrm{H}), 7.51-7.49(\mathrm{~m}, 3$ $\mathrm{H}), 7.44-7.42(\mathrm{~m}, 4 \mathrm{H}), 7.38-7.36(\mathrm{~m}, 1 \mathrm{H}), 6.77(\mathrm{~s}, 1 \mathrm{H}), 4.86(\mathrm{~s}, 1 \mathrm{H})$, $4.21(\mathrm{~d}, J=17.0 \mathrm{~Hz}, 1 \mathrm{H}), 4.09-3.99(\mathrm{~m}, 3 \mathrm{H}), 3.04(\mathrm{~s}, 3 \mathrm{H})$.

${ }^{13} \mathrm{C}$ NMR $\left(126 \mathrm{MHz}, \mathrm{MeCN}-d_{3}\right): \delta=173.0,169.0,168.8,146.1,139.4$, $131.3,129.8,129.8,129.8,107.1,65.1,64.9,47.9$.

${ }^{11} \mathrm{~B}$ NMR $\left(128 \mathrm{MHz}, \mathrm{MeCN}-d_{3}\right): \delta=10.6$.

HRMS (ESI): $m / z$ [M - H] $]^{-}$calcd for $\mathrm{C}_{22} \mathrm{H}_{19} \mathrm{BN}_{3} \mathrm{O}_{6}$ : 431.1409; found: 431.1413.

\section{2-(MIDA-Boryl)-2-(5-phenyl-1H-pyrazol-1-yl)acetic Acid (5d)}

The reaction was carried out according to the general procedure using (E)-3-(dimethylamino)-1-phenylprop-2-en-1-one (18.4, $0.105 \mathrm{mmol}$, 1.05 equiv) at rt and purified by reversed-phase column chromatography to afford 5d (33.9 mg, $0.095 \mathrm{mmol}, 95 \%$ ) as a colorless solid; mp $165-168{ }^{\circ} \mathrm{C}$.

IR (neat): 3029, 2799, 2475, 1769, 1670, $1553 \mathrm{~cm}^{-1}$.

${ }^{1} \mathrm{H}$ NMR $\left(500 \mathrm{MHz}, \mathrm{MeCN}-d_{3}\right): \delta=7.52(\mathrm{~d}, J=2.0 \mathrm{~Hz}, 1 \mathrm{H}), 7.49-7.46$ (m, $3 \mathrm{H}), 7.38-7.36(\mathrm{~m}, 2 \mathrm{H}), 6.36(\mathrm{~d}, J=1.5 \mathrm{~Hz}, 1 \mathrm{H}), 4.86(\mathrm{~s}, 1 \mathrm{H}), 4.28$ (d, J = 17.0 Hz, $1 \mathrm{H}), 4.09-3.99(\mathrm{~m}, 3 \mathrm{H}), 3.04(\mathrm{~s}, 3 \mathrm{H})$.

${ }^{13} \mathrm{C}$ NMR $\left(126 \mathrm{MHz}, \mathrm{MeCN}-d_{3}\right): \delta=173.0,169.0,168.7,148.1,139.4$, $131.3,129.8,129.8,129.8,107.1,65.1,64.9,47.9$.

${ }^{11} \mathrm{~B}$ NMR $\left(128 \mathrm{MHz}, \mathrm{MeCN}-d_{3}\right): \delta=10.6$.

HRMS (ESI): $m / z$ [M - H] $]^{-}$calcd for $\mathrm{C}_{16} \mathrm{H}_{15} \mathrm{BN}_{3} \mathrm{O}_{6}$ : 355.1096; found: 355.1099 .

\section{2-[5-(1H-Pyrrol-2-yl)-1H-pyrazol-1-yl]-2-(MIDA-boryl)acetic Acid (5e)}

The reaction was carried out according to the general procedure using (E)-3-(dimethylamino)-1-(1H-pyrrol-2-yl)prop-2-en-1-one (18.1 mg, $0.11 \mathrm{mmol}, 1.1$ equiv) at $\mathrm{rt}$ and purified by reversed-phase column chromatography to afford $\mathbf{5 e}(33.9 \mathrm{mg}, 0.098 \mathrm{mmol}, 98 \%)$ as a brown solid; mp $170-175^{\circ} \mathrm{C}$ (decomp.).

IR (neat): 3374, 3146, 2982, 2262, 1748, 1607, $1511 \mathrm{~cm}^{-1}$.

${ }^{1} \mathrm{H}$ NMR (500 MHz, MeCN- $\left.d_{3}\right): \delta=9.51$ (br s), $7.47(\mathrm{~d}, J=2.0 \mathrm{~Hz}, 1 \mathrm{H}$ ), 6.90-6.89 (m, $1 \mathrm{H}), 6.38$ (d, J = 2.0 Hz, $1 \mathrm{H}), 6.27-6.25(\mathrm{~m}, 1 \mathrm{H}), 6.24-$ $6.23(\mathrm{~m}, 1 \mathrm{H}), 5.06(\mathrm{~s}, 1 \mathrm{H}), 4.29$ (d, J = $16.5 \mathrm{~Hz}, 1 \mathrm{H}), 4.10-4.01(\mathrm{~m}, 3$ $\mathrm{H}), 3.02(\mathrm{~s}, 3 \mathrm{H})$.

${ }^{13} \mathrm{C}$ NMR $\left(126 \mathrm{MHz}, \mathrm{MeCN}-d_{3}\right): \delta=173.0,169.1,168.9,139.3,138.8$, $121.3,120.7,110.2,109.1,105.1,65.1,65.0,47.9$.

${ }^{11} \mathrm{~B}$ NMR $\left(128 \mathrm{MHz}, \mathrm{MeCN}-d_{3}\right): \delta=10.6$.

HRMS (ESI): $m / z$ [M $-\mathrm{H}]^{-}$calcd for $\mathrm{C}_{14} \mathrm{H}_{14} \mathrm{BN}_{4} \mathrm{O}_{6}$ : 344.1048; found: 344.1052 . 
2-[5-(Furan-2-yl)-1H-pyrazol-1-yl]-2-(MIDA-boryl)acetic Acid (5f) The reaction was carried out according to the general procedure using (E)-3-(dimethylamino)-1-(furan-2-yl)prop-2-en-1-one (18.2 mg, 0.11 mmol, 1.1 equiv) at $\mathrm{rt}$ and purified by reversed-phase column chromatography to afford $\mathbf{5 f}$ ( $32.6 \mathrm{mg}, 0.094 \mathrm{mmol}, 94 \%$ ) as a brown solid; mp $140-142{ }^{\circ} \mathrm{C}$ (decomp.).

IR (neat): 2982, 2886, 1746, $1527 \mathrm{~cm}^{-1}$.

${ }^{1} \mathrm{H}$ NMR $\left(500 \mathrm{MHz}, \mathrm{MeCN}-d_{3}\right): \delta=7.60(\mathrm{dd}, J=1.9,1.0 \mathrm{~Hz}, 1 \mathrm{H}), 7.49$ $(\mathrm{d}, J=2.0 \mathrm{~Hz}, 1 \mathrm{H}), 6.67(\mathrm{dd}, J=3.5,1.0 \mathrm{~Hz}, 1 \mathrm{H}), 6.56(\mathrm{dd}, J=3.5,2.0$ $\mathrm{Hz}, 1 \mathrm{H}), 6.55$ (d, J = 2.0 Hz, $1 \mathrm{H}), 5.26(\mathrm{~s}, 1 \mathrm{H}), 4.31$ (d, J = $16.5 \mathrm{~Hz}, 1 \mathrm{H})$, 4.11-4.02 (m, $3 \mathrm{H}), 3.05$ (s, $3 \mathrm{H})$.

${ }^{13} \mathrm{C}$ NMR $\left(126 \mathrm{MHz}, \mathrm{MeCN}-d_{3}\right): \delta=172.9,169.0,168.9,145.2,144.3$, 139.5, 136.3, 112.5, 110.1, 106.0, 65.2, 65.1, 48.0.

${ }^{11} \mathrm{~B}$ NMR $\left(128 \mathrm{MHz}, \mathrm{MeCN}-d_{3}\right): \delta=10.7$.

HRMS (ESI): $m / z$ [M - H] $]^{-}$calcd for $\mathrm{C}_{14} \mathrm{H}_{13} \mathrm{BN}_{3} \mathrm{O}_{7}$ : 345.0888; found: 345.0894 .

\section{2-(MIDA-Boryl)-2-(4-phenyl-1H-pyrazol-1-yl)acetic Acid (5g)}

The reaction was carried out on a 1-mmol scale according to the general procedure using 2-phenylmalonaldehyde (156 mg, $1.05 \mathrm{mmol}$, 1.05 equiv) at rt and purified by reversed-phase column chromatography to afford $\mathbf{5 g}$ (294 $\mathrm{mg}, 0.82 \mathrm{mmol}, 82 \%)$ as a colorless solid; $\mathrm{mp}$ $150-152{ }^{\circ} \mathrm{C}$ (decomp.).

IR (neat): 3000, 2981, 1757, 1729, 1608, $1570 \mathrm{~cm}^{-1}$.

${ }^{1} \mathrm{H}$ NMR $\left(500 \mathrm{MHz}, \mathrm{MeCN}-d_{3}\right): \delta=7.88(\mathrm{~s}, 1 \mathrm{H}$ ), 7.80 (s, $1 \mathrm{H}$ ), 7.55-7.54 (m, 2 H), 7.38-7.35 (m, 2 H), 7.24-7.22 (m, 1 H), $5.04(\mathrm{~s}, 1 \mathrm{H}), 4.21$ (d, $J=17.0 \mathrm{~Hz}, 1 \mathrm{H}), 4.08(\mathrm{~d}, J=17.0 \mathrm{~Hz}, 1 \mathrm{H}), 4.04(\mathrm{~d}, J=17.0 \mathrm{~Hz}, 1 \mathrm{H})$, $3.95(\mathrm{~d}, J=17.0 \mathrm{~Hz}, 1 \mathrm{H}), 3.11(\mathrm{~s}, 3 \mathrm{H})$.

${ }^{13} \mathrm{C}$ NMR $\left(126 \mathrm{MHz}, \mathrm{MeCN}-d_{3}\right): \delta=172.8,168.8,168.6,137.0,133.4$, $129.8,129.8,127.3,126.2,123.8,64.8,64.5,47.9$.

${ }^{11} \mathrm{~B}$ NMR $\left(128 \mathrm{MHz}, \mathrm{MeCN}-d_{3}\right): \delta=10.5$.

HRMS (ESI): $m / z$ [M-H] $]^{-}$calcd for $\mathrm{C}_{16} \mathrm{H}_{15} \mathrm{BN}_{3} \mathrm{O}_{6}$ : 355.1096; found: 355.1100 .

\section{2-[4-(Biphenyl-4-yl)-1H-pyrazol-1-yl]-2-(MIDA-boryl)acetic Acid (5h)}

The reaction was carried out according to the general procedure using 2-(biphenyl-4-yl)malonaldehyde ( $24.7 \mathrm{mg}, 0.11 \mathrm{mmol}, 1.1$ equiv) at $\mathrm{rt}$ and purified by reversed-phase column chromatography to afford $\mathbf{5 h}$ (27.7 $\mathrm{mg}, 0.064 \mathrm{mmol}, 64 \%$ ) as a colorless solid; mp $213-215^{\circ} \mathrm{C}$ (decomp.).

IR (neat): 3034, 2964, 1769, 1716, 1615, $1575 \mathrm{~cm}^{-1}$.

${ }^{1} \mathrm{H}$ NMR (500 MHz, DMSO- $d_{6}$ ): $\delta=8.07(\mathrm{~s}, 1 \mathrm{H}), 7.91(\mathrm{~s}, 1 \mathrm{H}), 7.70-$ $7.64(\mathrm{~m}, 6 \mathrm{H}), 7.46(\mathrm{t}, J=7.5 \mathrm{~Hz}, 2 \mathrm{H}), 7.35(\mathrm{t}, J=7.5 \mathrm{~Hz}, 1 \mathrm{H}), 5.09(\mathrm{~s}, 1$ $\mathrm{H}), 4.45(\mathrm{~d}, J=17.0 \mathrm{~Hz}, 1 \mathrm{H}), 4.32(\mathrm{~d}, J=17.0 \mathrm{~Hz}, 1 \mathrm{H}), 4.18(\mathrm{~d}, J=17.0$ $\mathrm{Hz}, 1 \mathrm{H}), 4.05$ (d, J = $17.0 \mathrm{~Hz}, 1 \mathrm{H}), 3.08$ (s, $3 \mathrm{H}$ ).

${ }^{13} \mathrm{C}$ NMR $\left(126 \mathrm{MHz}\right.$, DMSO- $\left.d_{6}\right): \delta=172.6,168.8,168.3,139.8,137.5$, 135.8, 131.7, 128.8, 127.2, 127.1, 126.3, 125.4, 121.2, 63.5, 63.2, 46.9.

${ }^{11} \mathrm{~B}$ NMR (128 MHz, DMSO- $\left.d_{6}\right): \delta=11.2$.

HRMS (ESI): $m / z$ [M - H] $]^{-}$calcd for $\mathrm{C}_{22} \mathrm{H}_{19} \mathrm{BN}_{3} \mathrm{O}_{6}$ : 431.1409; found: 431.1399 .

2-(MIDA-Boryl)-2-(3-methyl-5-oxo-2,5-dihydro-1H-pyrazol-1yl)acetic Acid-Trifluoroacetic Acid (5i)

The reaction was carried out according to the general procedure using ethyl acetoacetate ( $10.8 \mu \mathrm{L}, 0.1 \mathrm{mmol}, 1$ equiv) at rt and purified by filtration to afford $\mathbf{5 i}$ ( $28.4 \mathrm{mg}, 0.067 \mathrm{mmol}, 67 \%)$ as a colorless solid; mp $158-161^{\circ} \mathrm{C}$.

IR (neat): 3217, 2969, 2541, 1909, 1770. 1736, 1639, $1575 \mathrm{~cm}^{-1}$.

${ }^{1} \mathrm{H}$ NMR (500 MHz, DMSO- $d_{6}$ ): $\delta=5.36$ (br s, $1 \mathrm{H}$ ), 4.66 (br s, $1 \mathrm{H}$ ), 4.44 $(\mathrm{d}, J=17.0 \mathrm{~Hz}, 1 \mathrm{H}), 4.27(\mathrm{~d}, J=16.5 \mathrm{~Hz}, 1 \mathrm{H}), 4.16-4.11(\mathrm{~m}, 2 \mathrm{H}), 3.06$ (s, $3 \mathrm{H}), 2.10$ (s, $3 \mathrm{H})$.

${ }^{13} \mathrm{C}$ NMR $\left(126 \mathrm{MHz}\right.$, DMSO- $\left.d_{6}\right): \delta=172.8,172.0,168.8,168.4,158.3$ $(\mathrm{q}, J=36.6 \mathrm{~Hz}), 145.7,115.5(\mathrm{q}, J=292.8 \mathrm{~Hz}), 87.1,63.7,63.4,47.1$, 13.2.

${ }^{11} \mathrm{~B}$ NMR $\left(128 \mathrm{MHz}\right.$, DMSO- $\left.d_{6}\right): \delta=10.9$.

${ }^{19} \mathrm{~F} \mathrm{NMR}\left(376 \mathrm{MHz}, \mathrm{DMSO}-d_{6}\right): \delta=-74.7$.

HRMS (ESI): $m / z$ [M - $\left.\mathrm{CF}_{3} \mathrm{CO}_{2} \mathrm{H}_{2}\right]^{-}$calcd for $\mathrm{C}_{11} \mathrm{H}_{13} \mathrm{BN}_{3} \mathrm{O}_{7}$ : 309.0888; found: 309.0891 .

\section{2-(MIDA-Boryl)-2-(1-oxophthalazin-2(1H)-yl)acetic Acid (5j)}

The reaction was carried out according to the general procedure using methyl 2-formylbenzoate ( $18.1 \mathrm{mg}, 0.11 \mathrm{mmol}, 1.1$ equiv) at $\mathrm{rt}$ and purified by filtration to afford $\mathbf{5 j}$ ( $28.1 \mathrm{mg}, 0.078 \mathrm{mmol}, 78 \%)$ as a colorless solid; $\mathrm{mp} 235-236^{\circ} \mathrm{C}$ (decomp.).

IR (neat): 3072, 3013, 2970, 2908, 1753, 1726, 1624, $1574 \mathrm{~cm}^{-1}$.

${ }^{1} \mathrm{H}$ NMR (400 MHz, DMSO- $\left.d_{6}\right): \delta=12.9(\mathrm{~s}, 1 \mathrm{H}), 8.45$ (s, $\left.1 \mathrm{H}\right), 8.26$ (dd, $J=7.8 \mathrm{~Hz}, 1 \mathrm{H}), 7.97-7.96(\mathrm{~m}, 2 \mathrm{H}), 7.91-7.87(\mathrm{~m}, 1 \mathrm{H}), 5.39(\mathrm{~s}, 1 \mathrm{H})$, $4.50(\mathrm{~d}, J=17.2 \mathrm{~Hz}, 1 \mathrm{H}), 4.28(\mathrm{~d}, J=16.8 \mathrm{~Hz}, 1 \mathrm{H}), 4.20-4.14(\mathrm{~m}, 2 \mathrm{H})$, $3.13(\mathrm{~s}, 3 \mathrm{H})$.

${ }^{13} \mathrm{C}$ NMR $\left(126 \mathrm{MHz}, \mathrm{DMSO}-d_{6}\right): \delta=172.7,169.0,168.6,158.8,137.2$, 133.7, 132.1, 129.2, 126.8, 126.8, 125.9, 63.8, 63.5, 47.7.

${ }^{11} \mathrm{~B}$ NMR $\left(128 \mathrm{MHz}\right.$, DMSO- $\left.d_{6}\right): \delta=11.3$.

HRMS (ESI): $m / z$ [M - H] $]^{-}$calcd for $\mathrm{C}_{15} \mathrm{H}_{13} \mathrm{BN}_{3} \mathrm{O}_{7}$ : 357.0888; found: 357.0891 .

\section{2-(1-Hydroxybenzo[d][1,2,3]diazaborinin-2(1H)-yl)-2-(MIDA-bor- yl)acetic Acid-Trifluoroacetic Acid (5k)}

The reaction was carried out according to the general procedure using 2-formylphenylboronic acid ( $15.0 \mathrm{mg}, 0.1 \mathrm{mmol}, 1$ equiv) at $\mathrm{rt}$ and purified by filtration to afford $\mathbf{5 k}(23.2 \mathrm{mg}, 0.049 \mathrm{mmol}, 49 \%)$ as a colorless solid; $\mathrm{mp}>250{ }^{\circ} \mathrm{C}$.

IR (neat): 3312, 2982, 1780, 1754. 1722, 1674, $1524 \mathrm{~cm}^{-1}$.

${ }^{1} \mathrm{H}$ NMR $\left(500 \mathrm{MHz}\right.$, DMSO- $\left.d_{6}\right): \delta=8.26(\mathrm{~d}, J=7.5 \mathrm{~Hz}, 1 \mathrm{H}), 7.99(\mathrm{~s}, 3$ H), 7.72-7.71 (m, $2 \mathrm{H}), 7.61-7.58(\mathrm{~m}, 1 \mathrm{H}), 4.84(\mathrm{~s}, 1 \mathrm{H}), 4.46(\mathrm{~d}, J=$ $17.5 \mathrm{~Hz}, 1 \mathrm{H}), 4.20-4.11$ (m, $3 \mathrm{H}), 3.12(\mathrm{~s}, 3 \mathrm{H})$.

${ }^{13} \mathrm{C}$ NMR $\left(126 \mathrm{MHz}\right.$, DMSO- $\left.d_{6}\right): \delta=175.2,169.2,168.6,158.3(\mathrm{q}, J=$ $38.1 \mathrm{~Hz}$ ), 136.8, 135.2, 131.2, 130.9, 128.4, 126.6, 115.1 (q, $J=289.9$ $\mathrm{Hz}), 63.7,63.6,47.3$.

${ }^{11} \mathrm{~B}$ NMR $\left(128 \mathrm{MHz}, \mathrm{DMSO}-d_{6}\right): \delta=29.0,10.3$.

${ }^{19} \mathrm{~F}$ NMR (376 MHz, DMSO- $\left.d_{6}\right): \delta=-74.8$.

HRMS (ESI): $m / z$ [M - $\left.\mathrm{CF}_{3} \mathrm{CO}_{2} \mathrm{H}_{2}\right]^{-}$calcd for $\mathrm{C}_{14} \mathrm{H}_{14} \mathrm{~B}_{2} \mathrm{~N}_{3} \mathrm{O}_{7}$ : 356.1096; found: 356.1102 .

\section{2-[1,4-Dioxo-3,4-dihydrophthalazin-2(1H)-yl]-2-(MIDA-boryl)ace- tic Acid (51)}

The reaction was carried out according to the general procedure using phthalic anhydride ( $16.3 \mathrm{mg}, 0.11 \mathrm{mmol}, 1.1$ equiv) at $50{ }^{\circ} \mathrm{C}$ and purified by reversed-phase column chromatography to afford $\mathbf{5 1}(6.6 \mathrm{mg}$, $0.018 \mathrm{mmol}, 18 \%$ ) as a colorless solid; $\mathrm{mp} 185-190{ }^{\circ} \mathrm{C}$.

IR (neat): 2983, 1751, 1645, 1571, $1500 \mathrm{~cm}^{-1}$. 
${ }^{1} \mathrm{H}$ NMR $\left(500 \mathrm{MHz}, \mathrm{MeCN}-d_{3}\right): \delta=8.31-8.29(\mathrm{~m}, 1 \mathrm{H}), 7.96-7.95(\mathrm{~m}, 1$ H), 7.88-7.85 (m, $2 \mathrm{H}), 5.57(\mathrm{~s}, 1 \mathrm{H}), 4.26(\mathrm{~d}, J=17.0 \mathrm{~Hz}, 1 \mathrm{H}), 4.12(\mathrm{~d}$, $J=17.5 \mathrm{~Hz}, 1 \mathrm{H}), 4.06(\mathrm{~d}, J=17.0 \mathrm{~Hz}, 1 \mathrm{H}), 4.02(\mathrm{~d}, J=17.5 \mathrm{~Hz}, 1 \mathrm{H})$, $3.23(\mathrm{~s}, 3 \mathrm{H})$.

${ }^{13} \mathrm{C}$ NMR $\left(126 \mathrm{MHz}, \mathrm{MeCN}-d_{3}\right): \delta=173.5,169.1,169.0,159.6,150.4$, 134.2, 133.5, 129.7, 127.8, 125.2, 125.1, 65.1, 64.5, 48.6.

${ }^{11} \mathrm{~B}$ NMR $\left(128 \mathrm{MHz}, \mathrm{MeCN}-d_{3}\right): \delta=10.9$.

HRMS (ESI): $m / z$ [M - H] $]^{-}$calcd for $\mathrm{C}_{15} \mathrm{H}_{13} \mathrm{BN}_{3} \mathrm{O}_{8}$ : 373.0838; found: 373.0837.

\section{2-[(1,3-Dioxoisoindolin-2-yl)amino]-2-(MIDA-boryl)acetic Acid $\left(5 \mathbf{l}^{\prime}\right)$}

The reaction was carried out according to the general procedure using phthalic anhydride ( $16.3 \mathrm{mg}, 0.11 \mathrm{mmol}, 1.1$ equiv) at $50{ }^{\circ} \mathrm{C}$ and purified by reversed-phase column chromatography to afford $\mathbf{5 l}^{\prime}$ ( $13.7 \mathrm{mg}$, $0.037 \mathrm{mmol}, 37 \%$ ) as a colorless solid; $\mathrm{mp}>250^{\circ} \mathrm{C}$.

IR (neat): 2972, 1746, 1639, 1544, $1500 \mathrm{~cm}^{-1}$.

${ }^{1} \mathrm{H}$ NMR $\left(500 \mathrm{MHz}, \mathrm{MeCN}-d_{3}\right): \delta=7.84-7.79(\mathrm{~m}, 4 \mathrm{H}), 5.21(\mathrm{br} \mathrm{s}, 1 \mathrm{H})$, $4.23(\mathrm{~d}, J=17.0 \mathrm{~Hz}, 1 \mathrm{H}), 4.10(\mathrm{~d}, J=17.0 \mathrm{~Hz}, 1 \mathrm{H}), 4.05(\mathrm{~d}, J=17.5 \mathrm{~Hz}$, $1 \mathrm{H}$ ), 3.94 (d, J = 17.0 Hz, $1 \mathrm{H}$ ), 3.69 (br s, $1 \mathrm{H}$ ), 3.17 (s, $3 \mathrm{H}$ ).

${ }^{13} \mathrm{C}$ NMR $\left(126 \mathrm{MHz}, \mathrm{MeCN}-d_{3}\right): \delta=175.2,168.6,168.4,167.8,135.5$, 131.2, 124.1, 63.8, 63.5, 47.6.

${ }^{11} \mathrm{~B}$ NMR $\left(128 \mathrm{MHz}, \mathrm{MeCN}-d_{3}\right): \delta=9.8$

HRMS (ESI): $m / z$ [M $-\mathrm{H}]^{-}$calcd for $\mathrm{C}_{15} \mathrm{H}_{13} \mathrm{BN}_{3} \mathrm{O}_{8}$ : 373.0838; found: 373.0831.

\section{2-[1,2-Bis(tert-butoxycarbonyl)hydrazinyl]-2-(MIDA-boryl)acetic Acid (6a)}

To a stirred suspension of $\mathbf{1}$ ( $44.5 \mathrm{mg}, 0.1 \mathrm{mmol}, 1$ equiv) in THF $(0.35$ $\mathrm{mL})$ and $\mathrm{MeOH}(0.15 \mathrm{~mL})$ at $0{ }^{\circ} \mathrm{C}$ was added $\mathrm{TMSCHN}_{2}$ ( $2 \mathrm{M}$ in hexane, $0.1 \mathrm{~mL}, 0.2 \mathrm{mmol}, 2$ equiv). The mixture was stirred for $30 \mathrm{~min}$ at $0{ }^{\circ} \mathrm{C}$. After addition of $\mathrm{AcOH}(0.1 \mathrm{~mL})$, the mixture was loaded onto Celite, then the volatiles were removed in vacuo. The residue was subjected to normal-phase column chromatography to afford $\mathbf{6 a}$ ( $38.5 \mathrm{mg}, 0.084$ $\mathrm{mmol}, 84 \%$ ) as a colorless solid; $\mathrm{mp} 115-117^{\circ} \mathrm{C}$.

IR (neat): 2983, 1760, $1713 \mathrm{~cm}^{-1}$.

${ }^{1} \mathrm{H}$ NMR (500 MHz, MeCN-d $d_{3}$ ): $\delta=6.97$ (br s, $1 \mathrm{H}$ ), 4.58 (br s, $1 \mathrm{H}$ ), 4.02-3.92 (m, $4 \mathrm{H}), 3.68$ (s, $3 \mathrm{H}), 3.19$ (s, $3 \mathrm{H}), 1.43-1.41$ (overlapped, $18 \mathrm{H})$.

${ }^{13} \mathrm{C}$ NMR $\left(126 \mathrm{MHz}, \mathrm{MeCN}-d_{3}\right): \delta=174.1,168.8,82.7,81.4,64.5,63.9$, 52.7, 47.6, 28.4, 28.2.

${ }^{11} \mathrm{~B}$ NMR $\left(128 \mathrm{MHz}, \mathrm{MeCN}-d_{3}\right): \delta=10.1$.

HRMS (ESI): $m / z[\mathrm{M}+\mathrm{Na}]^{+}$calcd for $\mathrm{C}_{18} \mathrm{H}_{30} \mathrm{BN}_{3} \mathrm{NaO}_{10}: 481.1953$; found: 481.1952.

\section{Di-tert-butyl 1-[2-Hydroxy-1-(MIDA-boryl)ethyl]hydrazine-1,2-di- carboxylate (6b)}

To a stirred suspension of $\mathbf{1}$ ( $44.5 \mathrm{mg}, 0.1 \mathrm{mmol}, 1$ equiv) and HATU ( $45.6 \mathrm{mg}, 0.12 \mathrm{mmol}, 1.2$ equiv) in MeCN $(1 \mathrm{~mL})$ was added DIPEA ( $34.8 \mu \mathrm{L}, 0.2 \mathrm{mmol}, 2$ equiv). The mixture was stirred at $\mathrm{rt}$ for $15 \mathrm{~min}$. $\mathrm{NaBH}_{4}$ (11.3 mg, $0.3 \mathrm{mmol}$, 3 equiv) was added to the solution, then the mixture was stirred at $\mathrm{rt}$ for $3 \mathrm{~h}$. After addition of $\mathrm{MeOH}(0.5 \mathrm{~mL})$, the mixture was loaded onto Celite, then the volatiles were removed in vacuo. The residue was subjected to reversed-phase column chromatography to afford $\mathbf{6 b}(28.3 \mathrm{mg}, 0.066 \mathrm{mmol}, 66 \%)$ as a colorless solid; $\mathrm{mp} 148-150{ }^{\circ} \mathrm{C}$.
IR (neat): 3411, 2981, 2962, 2906, 1768, $1705 \mathrm{~cm}^{-1}$.

${ }^{1} \mathrm{H}$ NMR $\left(500 \mathrm{MHz}, \mathrm{MeCN}-d_{3}\right): \delta=7.04-6.82(\mathrm{br}, 1 \mathrm{H}), 4.12-3.85(\mathrm{~m}, 5$ H), 3.65-3.53 (br, 2 H), 3.24-2.99 (br, 3 H), 1.43-1.40 (overlapped, 18 $\mathrm{H})$.

${ }^{13} \mathrm{C}$ NMR $\left(126 \mathrm{MHz}, \mathrm{MeCN}-d_{3}\right): \delta=169.0,168.7,82.1,81.4,63.9,63.0$, $60.2,47.9,46.8,28.4,28.4$.

${ }^{11} \mathrm{~B}$ NMR $\left(128 \mathrm{MHz}, \mathrm{MeCN}-d_{3}\right): \delta=10.6$.

HRMS (ESI): $m / z$ [M + Na] $]^{+}$calcd for $\mathrm{C}_{17} \mathrm{H}_{30} \mathrm{BN}_{3} \mathrm{NaO}_{9}$ : 453.2004; found: 453.1994 .

\section{Di-tert-butyl \{(MIDA-boryl)[5-(phenylamino)-1,3,4-oxadiazol-2- yl]methyl\}hydrazine-1,2-dicarboxylate (6c)}

To a stirred suspension of $\mathbf{1}$ ( $44.5 \mathrm{mg}, 0.1 \mathrm{mmol}, 1$ equiv) and HATU (41.8 mg, $0.11 \mathrm{mmol}, 1.1$ equiv) in $\mathrm{MeCN}(1 \mathrm{~mL})$ was added DIPEA ( $52.3 \mu \mathrm{L}, 0.3 \mathrm{mmol}, 3$ equiv). The mixture was stirred at $\mathrm{rt}$ for $15 \mathrm{~min}$. $\mathrm{N}$-Phenylhydrazinecarbothioamide ( $18.4 \mathrm{mg}, 0.11 \mathrm{mmol}, 1.1$ equiv) was added to the solution, then the mixture was stirred at $\mathrm{rt}$ for 30 min. $\mathrm{TsCl}$ ( $41.9 \mathrm{mg}, 0.22 \mathrm{mmol}, 2.2$ equiv) was added, then the mixture was stirred at rt for $4 \mathrm{~h}$. After $4 \mathrm{~h}$, another aliquot of TsCl (19.1 $\mathrm{mg}, 0.1 \mathrm{mmol}, 1$ equiv) and DIPEA ( $52.3 \mu \mathrm{L}, 0.3 \mathrm{mmol}, 3$ equiv) were added, then the mixture was stirred at rt for $20 \mathrm{~h}$. The mixture was loaded onto Celite, then the volatiles were removed in vacuo. The residue was subjected to reversed-phase column chromatography to afford $\mathbf{6 c}$ (38.2 mg, $0.068 \mathrm{mmol}, 68 \%$ ) as a colorless solid; mp 157-160 ${ }^{\circ} \mathrm{C}$.

IR (neat): 3328, 2982, 2936, 1778, 1740, 1700, $1515 \mathrm{~cm}^{-1}$.

${ }^{1} \mathrm{H}$ NMR $\left(500 \mathrm{MHz}, \mathrm{MeCN}-d_{3}\right): \delta=7.92-7.88(\mathrm{~m}, 4 \mathrm{H}), 7.17-7.02(\mathrm{br}, 1$ H), 5.10 (br s, 1 H), 4.14-3.99 (m, 4 H), 3.28 (s, $3 \mathrm{H}), 1.44$ (overlapped, $18 \mathrm{H})$.

${ }^{13} \mathrm{C}$ NMR $\left(126 \mathrm{MHz}, \mathrm{MeCN}-d_{3}\right): \delta=168.5,168.4,163.1,136.3,129.6$, $124.9,82.1,65.0,64.3,47.9,28.4,28.1$.

${ }^{11} \mathrm{~B}$ NMR $\left(128 \mathrm{MHz}, \mathrm{MeCN}-d_{3}\right): \delta=10.3$.

HRMS (ESI): $m / z[\mathrm{M}+\mathrm{Na}]^{+}$calcd for $\mathrm{C}_{25} \mathrm{H}_{31} \mathrm{BN}_{4} \mathrm{NaO}_{12}: 612.1960$; found: 612.1959 .

Di-tert-butyl 1-[1-(MIDA-boryl)-2-(4-nitrobenzyloxy)-2-oxoethyl]hydrazine-1,2-dicarboxylate (6d)

To a stirred suspension of $\mathbf{1}$ ( $44.5 \mathrm{mg}, 0.1 \mathrm{mmol}, 1$ equiv), 4-nitrobenzyl alcohol (18 mg, $0.12 \mathrm{mmol}, 1.2$ equiv), and DMAP (1.2 mg, 0.01 mmol, $10 \mathrm{~mol} \%)$ in DCM $(0.66 \mathrm{~mL})$ and $\mathrm{MeCN}(0.33 \mathrm{~mL})$ was added DIC ( $18.7 \mu \mathrm{L}, 0.12 \mathrm{mmol}, 1.2$ equiv). The mixture was stirred at $\mathrm{rt}$ for $20 \mathrm{~h}$. The mixture was filtered then loaded onto Celite, then the volatiles were removed in vacuo. The residue was subjected to normalphase column chromatography to afford $6 \mathbf{d}(52.7 \mathrm{mg}, 0.091 \mathrm{mmol}$, 91\%) as a colorless solid; $\mathrm{mp} 155-158^{\circ} \mathrm{C}$.

IR (neat): 3314, 2982, 2942, 1779, 1757, 1724, 1675, 1605, $1514 \mathrm{~cm}^{-1}$. ${ }^{1} \mathrm{H}$ NMR $\left(500 \mathrm{MHz}, \mathrm{MeCN}-d_{3}\right): \delta=8.21-8.19(\mathrm{~m}, 2 \mathrm{H}), 7.63(\mathrm{~d}, J=8.5$ $\mathrm{Hz}, 2 \mathrm{H}$ ), 7.03 (br s, $1 \mathrm{H}$ ), 5.33 (second order, $1 \mathrm{H}$ ), 5.25 (second order, $1 \mathrm{H}), 4.71$ (br, $1 \mathrm{H}), 4.05-3.93(\mathrm{~m}, 4 \mathrm{H}), 3.21(\mathrm{~s}, 3 \mathrm{H}), 1.42(\mathrm{~s}, 9 \mathrm{H}), 1.39$ (s, $9 \mathrm{H})$.

${ }^{13} \mathrm{C}$ NMR $\left(126 \mathrm{MHz}, \mathrm{MeCN}-d_{3}\right): \delta=173.5,168.7,168.7,148.6,144.7$, 129.3, 124.5, 81.6, 66.2, 64.6, 63.9, 47.6, 28.4, 28.2.

${ }^{11} \mathrm{~B}$ NMR $\left(128 \mathrm{MHz}, \mathrm{MeCN}-d_{3}\right): \delta=10.1$.

HRMS (ESI): $m / z[\mathrm{M}+\mathrm{Na}]^{+}$calcd for $\mathrm{C}_{24} \mathrm{H}_{33} \mathrm{BN}_{4} \mathrm{NaO}_{12}: 602.2117$; found: 602.2115 . 
Di-tert-butyl 1-\{2-[(1,3-Dioxoisoindolin-2-yl)oxy]-1-(MIDAboryl)-2-oxoethyl\}hydrazine-1,2-dicarboxylate (6e)

To a stirred suspension of $\mathbf{1}$ (1.2 g, $2.70 \mathrm{mmol}, 1$ equiv), $N$-hydroxyphthalimide (484 mg, $2.97 \mathrm{mmol}, 1.1$ equiv), and DMAP (33 mg, $0.27 \mathrm{mmol}, 10 \mathrm{~mol} \%)$ in $\mathrm{DCM}(20 \mathrm{~mL})$ and $\mathrm{MeCN}(7 \mathrm{~mL})$ was added DIC (0.464 mL, $2.97 \mathrm{mmol}, 1.1$ equiv). The mixture was stirred at $\mathrm{rt}$ for $14 \mathrm{~h}$. The mixture was filtered then loaded onto Celite, then the volatiles were removed in vacuo. The residue was subjected to normal-phase column chromatography to afford 6 e $(1.02 \mathrm{~g}, 1.73 \mathrm{mmol}$, $64 \%$ ) as a colorless solid; $\mathrm{mp} 155-158^{\circ} \mathrm{C}$.

IR (neat): 3328, 2982, 2936, 1778, 1740, 1700, $1515 \mathrm{~cm}^{-1}$.

${ }^{1} \mathrm{H}$ NMR (500 MHz, MeCN-d $d_{3}$ ): $\delta=7.92-7.88(\mathrm{~m}, 4 \mathrm{H}), 7.17-7.02(\mathrm{br}, 1$ H), 5.10 (br s, 1 H), 4.14-3.99 (m, 4 H), 3.28 (s, 3 H), 1.44 (overlapped, $18 \mathrm{H})$.

${ }^{13} \mathrm{C}$ NMR $\left(126 \mathrm{MHz}, \mathrm{MeCN}-d_{3}\right): \delta=168.5,168.4,163.1,136.3,129.6$, 124.9, 82.1, 65.0, 64.3, 47.9, 28.4, 28.1.

${ }^{11} \mathrm{~B}$ NMR $\left(128 \mathrm{MHz}, \mathrm{MeCN}-d_{3}\right): \delta=10.3$.

HRMS (ESI): $m / z[\mathrm{M}+\mathrm{Na}]^{+}$calcd for $\mathrm{C}_{25} \mathrm{H}_{31} \mathrm{BN}_{4} \mathrm{NaO}_{12}: 612.1960$; found: 612.1959 .

\section{Di-tert-butyl 3-(MIDA-boryl)-5-oxo-1,2,4-triazolidine-1,2-di- carboxylate (6f)}

To a stirred suspension of $\mathbf{1}$ ( $2.4 \mathrm{~g}, 5.39 \mathrm{mmol}, 1$ equiv) in $\mathrm{MeCN}$ (36 $\mathrm{mL}$ ) at $\mathrm{rt}$ was added DIPEA ( $2.07 \mathrm{~mL}, 11.86 \mathrm{mmol}, 2.2$ equiv) and DPPA (1.28 mL, $5.93 \mathrm{mmol}, 1.1$ equiv). The mixture was stirred at $50{ }^{\circ} \mathrm{C}$ for $1.5 \mathrm{~h}$. The mixture was cooled and loaded onto Celite, then the volatiles were removed in vacuo. The residue was subjected to normalphase column chromatography to afford $\mathbf{6 f}(1.53 \mathrm{~g}, 3.46 \mathrm{mmol}, 64 \%)$ as a colorless solid; mp $172-177^{\circ} \mathrm{C}$ (decomp.).

IR (neat): 3328, 2982, 2936, 1778, 1740, 1700, $1515 \mathrm{~cm}^{-1}$.

${ }^{1} \mathrm{H}$ NMR (500 MHz, MeCN-d $_{3}$ : $\delta=6.16$ (br s, $1 \mathrm{H}$ ), 5.05 (s, $1 \mathrm{H}$ ), 4.074.03 (m, 2 H), 3.94-3.88 (m, 2 H), 3.07 (s, 3 H), 1.47 (s, 9 H), 1.45 (s, 9 $\mathrm{H})$.

${ }^{13} \mathrm{C}$ NMR $\left(126 \mathrm{MHz}, \mathrm{MeCN}-d_{3}\right): \delta=168.7,167.9,158.8,153.9,84.6$, 83.7, 63.7, 63.4, 47.1, 28.2, 28.1.

${ }^{11} \mathrm{~B}$ NMR $\left(128 \mathrm{MHz}, \mathrm{MeCN}-d_{3}\right): \delta=8.9$.

HRMS (ESI): $m / z$ [M - H] $]^{-}$calcd for $\mathrm{C}_{17} \mathrm{H}_{26} \mathrm{BN}_{4} \mathrm{O}_{9}: 440.1835$; found: 440.1838 .

\section{Di-tert-butyl 3-(MIDA-boryl)-4-(4-nitrobenzyl)-5-oxo-1,2,4-tri-} azolidine-1,2-dicarboxylate $(6 \mathrm{~g})$

To a stirred suspension of $\mathbf{6 f}\left(100 \mathrm{mg}, 0.23 \mathrm{mmol}, 1\right.$ equiv), $\mathrm{K}_{2} \mathrm{CO}_{3}$ (63.6 mg, $46 \mathrm{mmol}, 2$ equiv), and TBAB $(7.3 \mathrm{mg}, 0.023 \mathrm{mmol}, 10$ $\mathrm{mol} \%$ ) in DMF ( $2 \mathrm{~mL}$ ) at rt was added 4-nitrobenzyl bromide $57.8 \mathrm{mg}$, $0.27 \mathrm{mmol}, 1.2$ equiv). The mixture was stirred at $60{ }^{\circ} \mathrm{C}$ for $20 \mathrm{~h}$. The mixture was cooled and loaded onto Celite, then the volatiles were removed in vacuo. The residue was subjected to normal-phase column chromatography. Upon removal of volatiles and trituration with $\mathrm{Et}_{2} \mathrm{O}$, $\mathbf{6 g}$ (70.2 mg, $0.12 \mathrm{mmol}, 53 \%$ ) was afforded as a colorless solid. Crystals suitable for X-ray diffraction were grown in $\mathrm{MeCN} / \mathrm{Et}_{2} \mathrm{O}$ using the vapor diffusion method; $\mathrm{mp} 195-200{ }^{\circ} \mathrm{C}$ (decomp.).

IR (neat): 3312, 2982, 1780, 1754, 1722, 1674, $1524 \mathrm{~cm}^{-1}$.

${ }^{1} \mathrm{H}$ NMR $\left(500 \mathrm{MHz}\right.$, DMSO- $\left.d_{6}\right): \delta=8.21(\mathrm{~d}, J=9.0 \mathrm{~Hz}, 2 \mathrm{H}), 7.44(\mathrm{~d}, J=$ $8.5 \mathrm{~Hz}, 2 \mathrm{H}), 5.07(\mathrm{~s}, 1 \mathrm{H}), 4.84(\mathrm{~d}, J=17.0 \mathrm{~Hz}, 1 \mathrm{H}), 4.40(\mathrm{~d}, J=17.5 \mathrm{~Hz}$, $1 \mathrm{H}), 4.34(\mathrm{~d}, J=17.0 \mathrm{~Hz}, 1 \mathrm{H}), 4.26(\mathrm{~d}, J=17.0 \mathrm{~Hz}, 1 \mathrm{H}), 4.08(\mathrm{~d}, J=17.0$ $\mathrm{Hz}, 1 \mathrm{H}), 3.92(\mathrm{~d}, J=17.0 \mathrm{~Hz}, 1 \mathrm{H}), 3.03(\mathrm{~s}, 3 \mathrm{H}), 1.42(\mathrm{~s}, 9 \mathrm{H}), 1.40(\mathrm{~s}, 9$ $\mathrm{H})$.
${ }^{13} \mathrm{C}$ NMR $\left(126 \mathrm{MHz}\right.$, DMSO- $\left.d_{6}\right): \delta=168.4,167.6,157.4,150.9,146.9$, $144.8,128.0,123.7,83.5,82.3,62.5,62.4,46.3,44.3,27.6,27.4$.

${ }^{11} \mathrm{~B}$ NMR $\left(128 \mathrm{MHz}, \mathrm{DMSO}-d_{6}\right): \delta=9.7$.

HRMS (ESI): $m / z[M+N a]^{+}$calcd for $\mathrm{C}_{24} \mathrm{H}_{32} \mathrm{BN}_{5} \mathrm{NaO}_{11}: 599.2120$; found: 599.2109 .

\section{Conflict of Interest}

The authors declare no conflict of interest.

\section{Funding Information}

The Natural Sciences and Engineering Research Council of Canada is gratefully acknowledged for their financial support. C.-H. T. would like to thank the Connaught Fund (University of Toronto).

\section{Acknowledgment}

Dr. Alan J. Lough (X-ray crystallography lab, University of Toronto) is thanked for X-ray structure determination of $\mathbf{6 g}$. Mr. Bijan Mirabi (Lautens lab, University of Toronto) is thanked for assistance with e.r. determination of $\mathbf{6 d}$ using chiral HPLC. The Taylor lab (University of Toronto) is thanked for FTIR instrument accommodation. Members of the Yudin lab are thanked for valuable discussions.

\section{Supporting Information}

Supporting information for this article is available online at https://doi.org/10.1055/s-0040-1706046.

\section{References}

(1) (a) Wu, X.; Chen, X.-X.; Fossey, J. S.; James, T. D.; Jiang, Y.-B. Chem. Soc. Rev. 2013, 42, 8032. (b) Dimitrijević, E.; Taylor, M. S. ACS Catal. 2013, 3, 945. (c) Li, J.; Ballmer, S. G.; Gillis, E. P.; Fujii, S.; Schmidt, M. J.; Palazzolo, A. M. E.; Lehmann, J. W.; Morehouse, G. F.; Burke, M. D. Science 2015, 347, 1221. (d) Smoum, R.; Rubinstein, A.; Dembitsky, V. M.; Srebnik, M. Chem. Rev. 2012, 112, 4156.

(2) Diaz, D. B.; Yudin, A. K. Nat. Chem. 2017, 9, 731.

(3) (a) Chen, D.; Frezza, M.; Schmitt, S.; Kanwar, J.; Dou, D.-P. Curr. Cancer Drug Targets 2011, 11, 239. (b) Adams, S.; Miller, G. T.; Jesson, M. I.; Watanabe, T.; Jones, B.; Wallner, B. P. Cancer Res. 2004, 64, 5471. (c) Seavey, M. M.; Lu, L. D.; Stump, K. L.; Wallace, N. H.; Ruggeri, B. A. Int. Immunopharmacol. 2012, 12, 257. (d) Zhanel, G. G.; Lawrence, C. K.; Adam, H.; Schweizer, F.; Zelenitsky, S.; Zhanel, M.; Lagacé-Wiens, P. R. S.; Walkty, A.; Denisuik, A.; Golden, A.; Gin, A. S.; Hoban, D. J.; Lynch, J. P. III.; Karlowsky, J. A. Drugs 2018, 78, 65. (e) Milo, L. J. Jr.; Lai, J. H.; Wu, W.; Liu, Y.; Maw, H.; Li, Y.; Jin, Z.; Shu, Y.; Poplawski, S. E.; Wu, D.; Sanford, D. G.; Sudmeier, J. L.; Bachovchin, W. W. J. Med. Chem. 2011, 54, 4365.

(4) (a) Andrés, P.; Ballano, G.; Calaza, I.; Cativiela, C. Chem. Soc. Rev. 2016, 45, 2291. (b) Beenen, M. A.; An, C.; Ellman, J. A. J. Am. Chem. Soc. 2008, 130, 6910. (c) Zajdlik, A.; Wang, Z.; Hickey, J. L.; Aman, A.; Schimmer, A. D.; Yudin, A. K. Angew. Chem. Int. Ed. 2013, 52, 8411. (d) Diaz, D. B.; Scully, C. C. G.; Liew, S. K.; Adachi, S.; Trinchera, P.; St. Denis, J. D.; Yudin, A. K. Angew. Chem. Int. Ed. 
2016, 55, 12659. (e) Shiro, T.; Schuhmacher, A.; Jackl, M. K.; Bode, J. W. Chem. Sci. 2018, 9, 5191. (f) Reyes, R. L.; Sato, M.; Iwai, T.; Sawamura, M. J. Am. Chem. Soc. 2020, 142, 589.

(5) Fustero, S.; Sánchez-Roselló, M.; Barrio, P.; Simón-Fuentes, A. Chem. Rev. 2011, 111, 6984.

(6) (a) Kerru, N.; Gummidi, L.; Maddila, S.; Gangu, K. K.; Jonnalagadda, S. B. Molecules 2020, 25, 1909. (b) Lamberth, C. Heterocycles 2007, 71, 1467. (c) Da Costa, L.; Scheers, E.; Coluccia, A.; Casulli, A.; Roche, M.; Di Giorgio, C.; Neyts, J.; Terme, T.; Cirilli, R.; La Regina, G.; Silvestri, R.; Mirabelli, C.; Vanelle, P. J. Med. Chem. 2018, 61, 8402.

(7) (a) Salaün, A.; Potel, M.; Roisnel, T.; Gall, P.; Le Grel, P. J. Org. Chem. 2005, 70, 6499. (b) Acherar, S.; Salaün, A.; Le Grel, P.; Le Grel, B.; Jamart-Grégnoire, B. Eur. J. Org. Chem. 2013, 2013, 5603. (c) Avan, I.; Hall, C. D.; Katritzky, A. R. Chem. Soc. Rev. 2014, 43, 3575. (d) Suć, J.; Tumir, L.-M.; Glavaš-Obrovac, L.; Jukic, M.; Piantanida, I.; Jerić, I. Org. Biomol. Chem. 2016, 14, 4865.

(8) (a) He, Z.; Yudin, A. K. J. Am. Chem. Soc. 2011, 133, 13770. (b) He, Z.; Trinchera, P.; Adachi, S.; St. Denis, J. D.; Yudin, A. K. Angew. Chem. Int. Ed. 2012, 51, 11092. (c) St. Denis, J. D.; Zajdlik, A.; Tan,
J.; Trinchera, P.; Lee, C. F.; He, Z.; Adachi, S.; Yudin, A. K. J. Am. Chem. Soc. 2014, 136, 17669. (d) Kaldas, S. J.; O'Keefe, K. T.; Mendoza-Sanchez, R.; Yudin, A. K. Chem. Eur. J. 2017, 23, 9711. (e) Holownia, A.; Tien, C.-H.; Diaz, D. B.; Larson, R. T.; Yudin, A. K. Angew. Chem. Int. Ed. 2019, 58, 15148.

(9) (a) List, B. J. Am. Chem. Soc. 2002, 124, 5656. (b) Bøgevig, A.; Kumaragurubaran, N.; Juhl, K.; Zhuang, W.; Jørgensen, K. A. Angew. Chem. Int. Ed. 2002, 41, 1790.

(10) Based on separation by HPLC on a chiral stationary phase column (see Supporting Information), the e.r. of $\mathbf{6 d}$, and thus $\mathbf{1}$ by correlation, was determined to be 60:40. Due to the almost racemic nature of 1, e.r. determination of derivatives $\mathbf{5}$ and $\mathbf{6}$ were not measured.

(11) Ivon, Y. M.; Mazurenko, I. V.; Kuchkovska, Y. O.; Voitenko, Z. V.; Grygorenko, O. O. Angew. Chem. Int. Ed. 2020, 59, 18016.

(12) Bindal, S.; Kumar, D.; Kommi, D. N.; Bhatiya, S.; Chakraborti, A. K. Synthesis 2011, 1930.

(13) Hou, T.-C.; Wu, Y.-Y.; Chiang, P.-Y.; Tan, K.-T. Chem. Sci. 2015, 6, 4643. 